\title{
Sequential Removal of Oppositely Charged Multiple Compounds from Water using Surface Modified Cellulose
}

\author{
Asma Batool and Suresh Valiyaveettil ${ }^{*}$ \\ Department of Chemistry, National University of Singapore, \\ 3 Science Drive 3, Singapore 117543 \\ *Corresponding author, E-mail address: chmsv@nus.edu.sg (S. Valiyaveettil)
}

Number of Pages: 6

Number of Tables:4

Number of Figures:3

\section{Table of Contents}

Figure S1. Images of banana stem, unmodified and PEI modified cellulose. (3)

Figure S2: Optical micrographs of unmodified and PEI modified cellulose. (3)

Figure S3: FTIR spectra of both adsorbents in the range of 700 to $4000 \mathrm{~cm}^{-1}$. (3)

Table S1: Chemical structure, physical and electrical properties of model pollutants. (2)

Table S2: Zeta potential and elemental analysis of adsorbents before and after successive adsorption of oppositely charged species from water. (4)

Table S3: Pseudo-first- order parameters for adsorption of AF, $\mathrm{Cu}(\mathrm{II})$, PTE and $\mathrm{Zn}(\mathrm{II})$. (4)

Table S4: Isotherm parameters for adsorption of AF, Cu(II), PTE and Zn(II). (5) 
Table S1. Chemical structure, physical and electrical properties of model pollutants.

\begin{tabular}{lllll}
\hline Pollutants & Chemical & $\begin{array}{l}\text { Molecular } \\
\text { Weight } \\
(\mathrm{g} / \mathrm{mol})\end{array}$ & $\begin{array}{l}\text { Ehysical } \\
\text { properties } \\
\& \mathrm{\Lambda}_{\max }\end{array}$ & $\begin{array}{l}\text { Electrical } \\
\text { properties }\end{array}$ \\
\hline Acid Fuchsin & Structures/Formula & Powder, Anionic & $546 \mathrm{~nm}$
\end{tabular}

Cupric

Sulphate

pentahydrate $\mathrm{CuSO}_{4} .5 \mathrm{H}_{2} \mathrm{O}$

$\begin{array}{ll}249.68 & \begin{array}{l}\text { Crystals } \\ \text { and lumps }\end{array} \quad \text { Cationic }\end{array}$

Perylene

tetracarboxy-

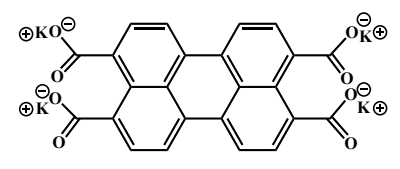

580.72

Solid, 466

$\mathrm{nm}$

Anionic

tetrapotassium

salt (PTE)

Zinc Acetate

$\mathrm{Zn}\left(\mathrm{CH}_{3} \mathrm{COO}\right)_{2} \cdot 2 \mathrm{H}_{2} \mathrm{O}$

219.51

Powder

Cationic

dihydrate 


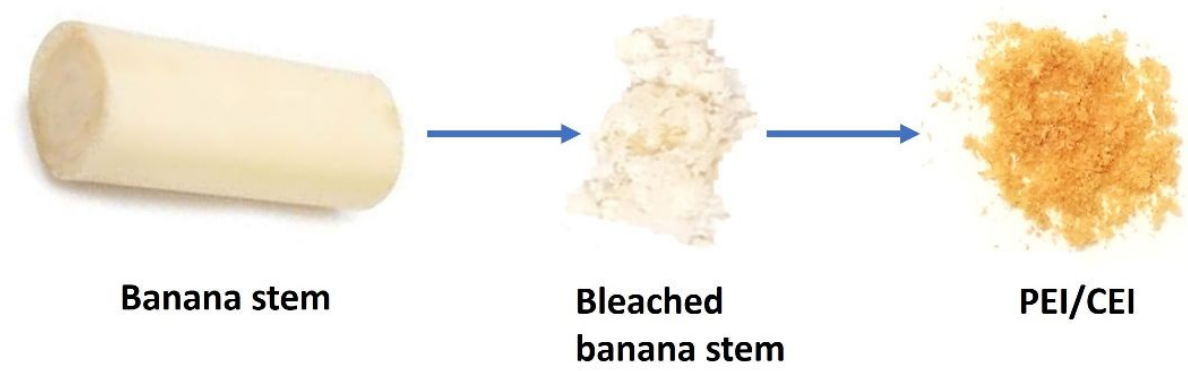

Figure S1. Images of banana stem, unmodified and PEI modified cellulose.
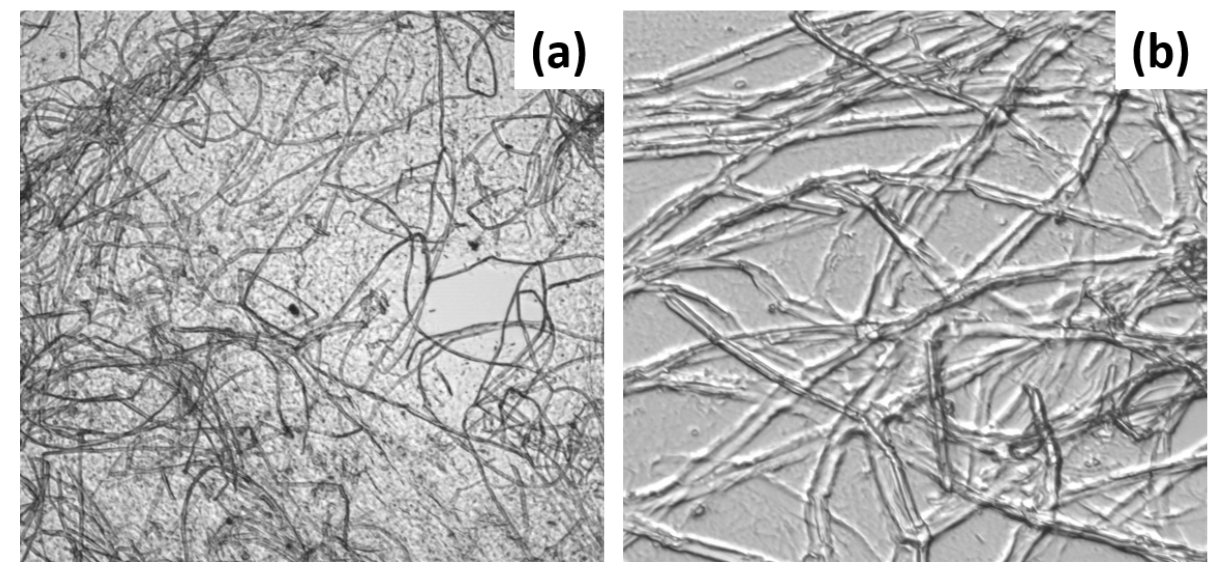

Figure S2. Optical micrographs of unmodified (a) and PEI modified (b) cellulose.

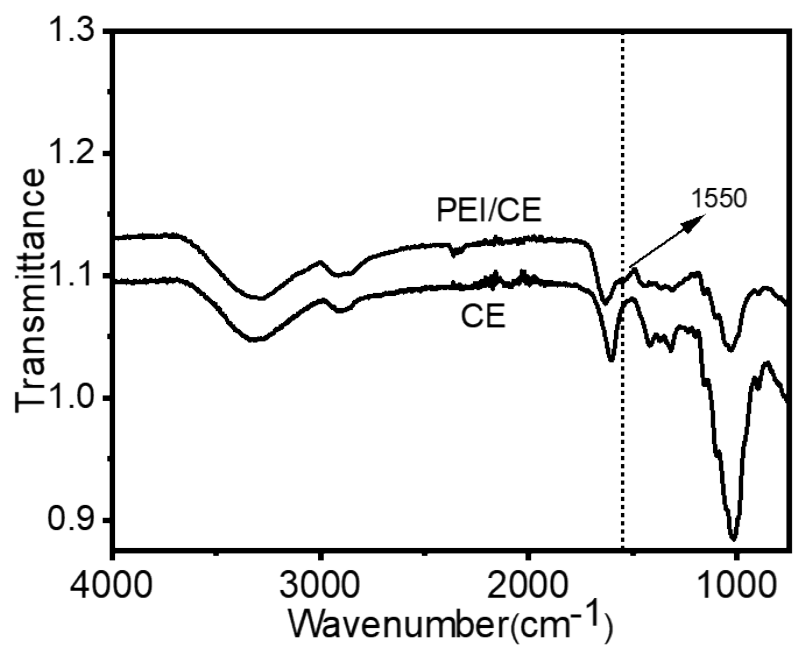

Figure S3. FTIR spectra of both adsorbents from spectral range of 700 to $4000 \mathrm{~cm}^{-1}$. 
Table S2: Zeta potential of adsorbents before and after successive adsorption of oppositely charged species from water.

\begin{tabular}{|l|l|l|l|}
\hline \multirow{2}{*}{ Materials } & \multirow{2}{*}{$\begin{array}{l}\text { Zeta } \\
\text { potentials(mV) }\end{array}$} & \multicolumn{2}{|l|}{ Elemental analysis } \\
\cline { 3 - 5 } & & $\begin{array}{c}\mathrm{Cu}(\mathrm{II}) \\
(\% \mathrm{Wt})\end{array}$ & $\begin{array}{l}\mathrm{Zn}(\mathrm{II}) \\
(\% \mathrm{Wt})\end{array}$ \\
\hline Bleached cellulose (CE) & -18 & - & - \\
\hline $\mathrm{PEI} / \mathrm{CE}$ & 21 & - & - \\
\hline $\mathrm{PEI} / \mathrm{CE} / \mathrm{AF}$ & -3 & - & - \\
\hline $\mathrm{PEI} / \mathrm{CE} / \mathrm{AF} / \mathrm{Cu}$ & 16.6 & 3.49 & - \\
\hline $\mathrm{PEI} / \mathrm{CE} / \mathrm{AF} / \mathrm{Cu} / \mathrm{PTE}$ & -13.5 & 3.38 & - \\
\hline $\mathrm{PEI} / \mathrm{CE} / \mathrm{AF} / \mathrm{Cu} / \mathrm{PTE} / \mathrm{Zn}$ & 11.3 & 3.20 & 2.21 \\
\hline
\end{tabular}

Table S3: Pseudo-first- order parameters for adsorption of AF, Cu(II), PTE and Zn(II).

\begin{tabular}{|c|c|c|c|c|c|c|c|c|c|c|c|c|}
\hline \multirow{3}{*}{$\begin{array}{l}\text { Conc } \\
\text { (mg/ } \\
\text { L) }\end{array}$} & \multicolumn{12}{|c|}{ Pseudo-First order model } \\
\hline & \multicolumn{3}{|c|}{$\mathbf{A F}$} & \multicolumn{3}{|c|}{$\mathrm{Cu}$ (II) } & \multicolumn{3}{|c|}{ PTE } & \multicolumn{3}{|c|}{$\mathbf{Z n}$} \\
\hline & Q & $\mathrm{k}_{1}$ & $\mathrm{R}^{2}$ & Q & $\mathrm{k}_{1}$ & $\mathrm{R}^{2}$ & Q & $\mathrm{k}_{1}$ & $\mathrm{R}^{2}$ & Q & $\mathrm{k}_{1}$ & $\mathrm{R}^{2}$ \\
\hline 50 & 1.942 & 0.007 & 0.126 & 4.026 & 0.007 & 0.144 & 0.463 & 0.015 & 0.121 & 11.43 & 0.009 & 0.474 \\
\hline 100 & 0.956 & 0.008 & 0.042 & 35.84 & 0.013 & 0.664 & 23.17 & 0.025 & 0.284 & 7.666 & 0.014 & 0.324 \\
\hline 200 & 8.167 & 0.012 & 0.268 & 23.16 & 0.012 & 0.394 & - & - & - & - & - & - \\
\hline 300 & 13.58 & 0.014 & 0.317 & 76.20 & 0.015 & 0.665 & - & - & - & - & - & - \\
\hline
\end{tabular}


Table S4: Isotherm parameters for adsorption of AF, $\mathrm{Cu}(\mathrm{II})$, PTE and $\mathrm{Zn}(\mathrm{II})$.

\begin{tabular}{|c|c|c|c|c|c|c|}
\hline \multirow[t]{2}{*}{$\begin{array}{l}\text { Dyes/Heavy } \\
\text { metals }\end{array}$} & \multicolumn{3}{|c|}{$\begin{array}{l}\text { Langmuir } \\
\text { Model }\end{array}$} & \multicolumn{3}{|c|}{$\begin{array}{l}\text { Freundlich } \\
\text { Model }\end{array}$} \\
\hline & $\overline{Q_{m}}$ & $\overline{K_{L}}$ & $\overline{\mathrm{R}^{2}}$ & $\mathrm{~K}_{\mathrm{F}}$ & $\mathrm{n}$ & $\overline{\mathrm{R}^{2}}$ \\
\hline $\mathrm{AF}$ & 93 & $1.075 \times 10^{-3}$ & 1 & 7.17 & -8.23 & 0.433 \\
\hline $\mathrm{Cu}(\mathrm{II})$ & 77 & -21.64 & 0.999 & 6.88 & -8.51 & 0.884 \\
\hline PTE & 56 & -18.1 & 0.996 & 4.86 & -2.11 & 0.960 \\
\hline $\mathrm{Zn}(\mathrm{II})$ & 38 & -0.299 & 0.95 & 9.58 & -2.085 & 0.874 \\
\hline
\end{tabular}

